\section{OPEN ACCESS}

Edited by:

Abbas Ghaderi,

Shiraz University of Medical Sciences,

Iran

Reviewed by:

Michael Hader

University Hospital Erlangen, Germany

Pavel Strnad,

University Hospital RWTH Aachen,

Germany

${ }^{*}$ Correspondence:

Raphael Mohr

raphael.mohr@charite.de

Specialty section:

This article was submitted to

Cancer Immunity and

Immunotherapy,

a section of the journal

Frontiers in Immunology

Received: 11 January 2021

Accepted: 15 March 2021

Published: 30 March 2021

Citation:

Mohr R, Jost-Brinkmann F, Özdirik B,

Lambrecht J, Hammerich L,

Loosen SH, Luedde T, Demir M,

Tacke $F$ and Roderburg C (2021)

Lessons From Immune

Checkpoint Inhibitor Trials in

Hepatocellular Carcinoma.

Front. Immunol. 12:652172.

doi: 10.3389/fimmu.2021.652172

\title{
Lessons From Immune Checkpoint Inhibitor Trials in Hepatocellular Carcinoma
}

\author{
Raphael Mohr ${ }^{1 *}$, Fabian Jost-Brinkmann ${ }^{1}$, Burcin Özdirik ${ }^{1}$, Joeri Lambrecht ${ }^{1}$, \\ Linda Hammerich ${ }^{1}$, Sven H. Loosen ${ }^{2}$, Tom Luedde ${ }^{2}$, Münevver Demir ${ }^{1}$, Frank Tacke ${ }^{1}$ \\ and Christoph Roderburg ${ }^{1,2}$
}

${ }^{1}$ Department of Hepatology and Gastroenterology, Charité University Medicine Berlin, Berlin, Germany, ${ }^{2}$ Clinic for Gastroenterology, Hepatology and Infectious Diseases, University Hospital Düsseldorf, Düsseldorf, Germany

The implementation of immune checkpoint inhibitors (ICI) into the clinical management of different malignancies has largely changed our understanding of cancer treatment. After having proven efficacy in different tumor entities such as malignant melanoma and lung cancer, $\mathrm{ICl}$ were intensively tested in the setting of hepatocellular carcinoma (HCC). Here they could achieve higher and more durable response rates compared to tyrosine-kinase inhibitors (TKI), that were sole standard of care for the last decade. Most recently, ICI treatment was approved in a first line setting of HCC, for cases not suitable for curative strategies. However, only a subset of patients benefits from $\mathrm{ICl}$ therapy, while others experience rapid tumor progression, worsening of liver function and poor prognosis. Efforts are being made to find immune characteristics that predict tumor responsiveness to $\mathrm{ICl}$, but no reliable biomarker could be identified so far. Nevertheless, data convincingly demonstrate that combination therapies (such as dual inhibition of PD-L1 and VEGF) are more effective than the application of single agents. In this review, we will briefly recapitulate the current algorithms for systemic treatment, discuss available results from checkpoint inhibitor trials and give an outlook on future directions of immunotherapy in HCC.

Keywords: hepatocellular carcinoma, immunotherapy, checkpoint inhibitor treatment, clinical trials, liver cirrhosis

\section{INTRODUCTION}

Hepatocellular carcinoma (HCC) is the fifth most common malignancy in men (7.9\% of all cancers) with 523,000 new cases per year worldwide and the seventh most common malignancy in women (6.5\% of all cancers) with 226,000 new cases $(1,2)$. Although the incidence and prevalence in western world countries is lower compared to Asia, HCC represents a major medical and socioeconomic problem worldwide, being one of the leading causes of cancer-related deaths $(2,3)$.

The vast majority of HCC arises in the context of liver cirrhosis, that means in a setting of chronic inflammation and continuous liver injury. By constant induction of cell death and compensatory hyperproliferation, but also via provoking an immunogen microenvironment, chronic inflammation leads to a pro-carcinogenic milieu (4). Following the prevalence of major risk factors for liver cirrhosis, the incidence of HCC has been steadily increasing over the last 
decades. It was only most recently that a reversal of this trend was observed in western world countries (5). The increase of HCC cases in the USA and Europe in the last decades has been mainly attributed to the hepatitis C epidemic in the 1970s and 1980s. Moreover, the fast-growing number of obesity and metabolic syndrome, leading to nonalcoholic fatty liver disease (NAFLD) and steatohepatitis (NASH), is likely to condition a future increase of liver cirrhosis and also HCC - despite the foreseeable decline of hepatitis C-related HCC. In a few countries (e.g., Thailand, Japan, Singapore) the HCC incidence could be stabilized or reduced by hepatitis B vaccination programs (6).

Despite significant advances in diagnosis and tumor therapy, the prognosis of HCC remains poor, especially in advanced stages. This is particularly due to the fact that HCC often occurs in functionally compromised livers or is only diagnosed when curative therapies such as resection, transplantation, or local ablative techniques are no longer possible. These patients are left to palliative treatment options only, including systemic tumor therapy. With the introduction of tyrosine-kinase inhibitors (TKI) and recently immune checkpoint inhibitors (ICI), pharmacological treatment options for patients with advanced HCC have greatly improved. Nevertheless, their efficacy is still not satisfying. Thus, there is an unmet need for novel treatment options to further improve patients' prognosis.

In this review, we will briefly recapitulate the current algorithms for systemic treatment, discuss available results from checkpoint inhibitor trials and give an outlook on future directions of immunotherapy in HCC.

\section{CURRENT AND EMERGING THERAPEUTIC OPTIONS FOR HCC}

Continuous viral (e.g., chronic hepatitis B, C, delta co-infection), toxic or metabolic liver injury leads to chronic liver inflammation and conditions the transformation toward fibrosis and cirrhosis. The proinflammatory environment of liver cirrhosis provides an ideal breeding ground for the development of hepatocellular carcinomas.

In this context, close surveillance for all patients with cirrhosis has been recommended in international guidelines. Nevertheless, numerous primary liver tumors are still diagnosed at tumor stages that are no longer curative (intermediate or advanced

\footnotetext{
Abbreviations: AFP, alpha-fetoprotein; BCLC, Barcelona Clinic of Liver Cancer; CTLA-4, cytotoxic T-lymphocyte-associated protein 4; DCR, disease control rate; DOR, duration of response; EORTC QLQ, European Organization for the Research and Treatment of Cancer Quality-of-Life Questionnaire; FGFR, fibroblast growth factor receptor; HCC, hepatocellular carcinoma; ICI, immune checkpoint inhibitors; irAE, immune related adverse events; LRT, locoregional therapies; NLR, neutrophil-lymphocyte ratio; NAFLD, nonalcoholic fatty liver disease; NASH, nonalcoholic steatohepatitis; ORR, objective response rate; OS, overall survival; PDGFR, platelet-derived growth factor receptors; PD-1, programmed cell death 1 protein; PD-L1, programmed death-ligand 1; PFS, progression free survival; TTD, time to deterioration; TTSD, time to symptom deterioration; TACE, transarterial chemoembolization; TKI, tyrosine-kinase inhibitors; VEGFR, vascular endothelial growth factor receptor.
}

stages of HCC according to the Barcelona Clinic of Liver Cancer (BCLC) staging system) (7). According to current guidelines these patients should be treated with systemic therapy. However, pharmacological treatment of HCC is challenging as HCCs show important tumor heterogeneity and arise from a distinct microenvironment, with regard to different etiologies of liver injury and different degrees of liver dysfunction. Considering the individual tumor microenvironment could be particularly relevant for immune-stimulating ICI strategies, as this might aggravate inflammatory and fibrogenic processes, e.g. in NASH (8).

HCC has long been considered to be refractory to systemic therapy. Trials with classical chemotherapy such as platinum derivatives or gemcitabine did not lead to a significant improvement in survival but proved to be very toxic against a background of impaired liver function. In 2008, the SHARP trial established sorafenib, which simultaneously inhibits tumor growth by targeting the Raf-MEK-ERK cascade as well as angiogenesis by targeting vascular endothelial growth factor (VEGFR) 2, plateletderived growth factor receptors (PDGFR) and KIT as a novel standard treatment in patients with advanced HCC (9). Although sorafenib showed greater efficacy in certain subgroups, such as patients with hepatitis $\mathrm{C}$ virus infection or elevated neutrophillymphocyte ratio (NLR), its overall moderate efficacy and poor toxicity profile limited its use in clinical practice $(10,11)$. In 2018 , lenvatinib, another TKI targeting VEGFR 1-3, fibroblast growth factor receptor (FGFR) 1-4, PDGFR, RET and KIT (12), was tested as non-inferior to sorafenib in the REFLECT trial and represented an alternative to the latter in the first line treatment of patients with advanced HCC or intermediate HCC refractory to loco-ablative treatments. Just recently, donafenib, another TKI was suggested as a third TKI suitable for first line therapy of HCC. In a phase II/III trial donafenib was associated with a longer median overall survival (OS) when compared with sorafenib (12.1 vs. 10.3 months, $\mathrm{p}=0.0363$ ), no significant differences were observed in the median progression free survival ( 3.7 vs. 3.6 months, $\mathrm{p}=0.2824$ ), objective response rate $(4.6 \%$ vs. $2.7 \%, p=0.2448)$, and disease control rate (30.8\% vs. $28.7 \%, \mathrm{p}=0.5532)(13)$.

OS with TKI treatment was approximately one year in both the SHARP and REFLECT trials, with a progression free survival (PFS) of approximately 4 months. After disease progression under a TKI, re-administration of a TKI was tested in the RESORCE study and the CELESTIAL study, with regorafenib and cabozantinib respectively. Both substances target the VEGFR 1-3, as well as the MET and AXL pathway (14), and have been approved for use in patients refractory to sorafenib $(15,16)$. In addition to these classical TKI, Ramucirumab, a novel antibody directed against VEGFR 2 has demonstrated efficacy when used in patients with elevated serum alpha-fetoprotein (AFP) levels (17). In summary, TKI built the standard-of-care treatment for patients with advanced HCC or intermediate stage HCC, refractory to, or unsuitable for loco-ablative treatments. However, moderate efficacy and unfavorable toxicities conditioned the need for better treatment options.

The introduction of ICI into the clinical management of different malignancies has changed our view on how to treat 
cancer. Immune checkpoints are "control points" of the immune system. They are based on surface receptors that, together with their ligands, prevent the immune system from attacking the body's own cells. In many malignant tumors, proteins that target immune checkpoints are upregulated. This allows the tumor cells to escape from attacks of the immune system (immune evasion). As shown in Figure 1, ICI block inhibitory immune checkpoints and thus trigger a defense response of the immune system toward tumor tissue. Immunotherapies seemed promising in patients with primary liver cancer, since cirrhotic livers feature an immunosuppressive environment that protect cancer cell from being recognized by the immune system, which, in turn, may be overcome by ICI (18). ICI were and are being tested in many different studies in the context of HCC.

\section{SINGLE-AGENT IMMUNOTHERAPY}

Immunotherapy has become a new and promising pillar in the treatment of HCC. So far mainly monoclonal antibodies inhibiting programmed cell death 1 protein (PD-1), programmed deathligand 1 (PD-L1), or cytotoxic T-lymphocyte-associated protein 4 (CTLA-4) were used in clinical trials of ICI.

Nivolumab blocks PD-1 and was tested in the setting of HCC in the non-comparative CheckMate 040 study (19). Patients with Child-Pugh A, pretreated with sorafenib $(n=182)$ or treatmentnaïve $(\mathrm{n}=80)$, received nivolumab in this phase I/II study in a dose-escalation (0.1-10 mg/kg every 2 weeks (Q2W)) and in a dose- expansion phase (3 $\mathrm{mg} / \mathrm{kg}$ Q2W). Primary endpoints were safety and tolerability for the escalation phase and objective response rate (ORR) for the expansion phase. ORR and disease control rate (DCR) were $20 \%$ and $64 \%$, respectively. $91 \%$ of responders had responses lasting 6 months or longer, and 55\% had responses lasting 12 months or longer. Median OS duration was 28.6 months in sorafenib naive patients and 15 months in patients pretreated with sorafenib. Additionally, a cohort of 49 patients with Child-Pugh B received a $240 \mathrm{mg}$ flat dose of nivolumab Q2W. Interestingly, the safety profile of nivolumab in these patients was comparable to that observed in patients with Child-Pugh A. In a Child-Pugh B setting, nivolumab monotherapy also demonstrated durable responses with ORR of $10 \%$ and DCR of $55 \%$ (20).

Based on that data, phase III CheckMate 459 study compared nivolumab $240 \mathrm{mg}$ Q2W $(\mathrm{n}=371)$ to sorafenib $(\mathrm{n}=372)$ in a first line setting. The differences in OS failed to meet statistical significance. The 33 -months OS was $29 \%$ for nivolumab vs. $21 \%$ for sorafenib (21). Nevertheless, overall improvements in median OS (16.4 vs. 14.7 months), ORR (15\% vs. 7\%, respectively), and CR rate ( $4 \%$ vs. $1 \%$, respectively) were considered clinically meaningful (22). The excellent survival in both arms is probably attributable to the subsequent therapy that patients received (49\% for nivolumab and 53\% for sorafenib, with $20 \%$ of patients treated with sorafenib receiving subsequent immunotherapy), which probably contributed to the study's negative results (23). Moreover, a slower deterioration of liver function as evidenced by albumin or bilirubin levels and ChildPugh scores was observed under nivolumab therapy.

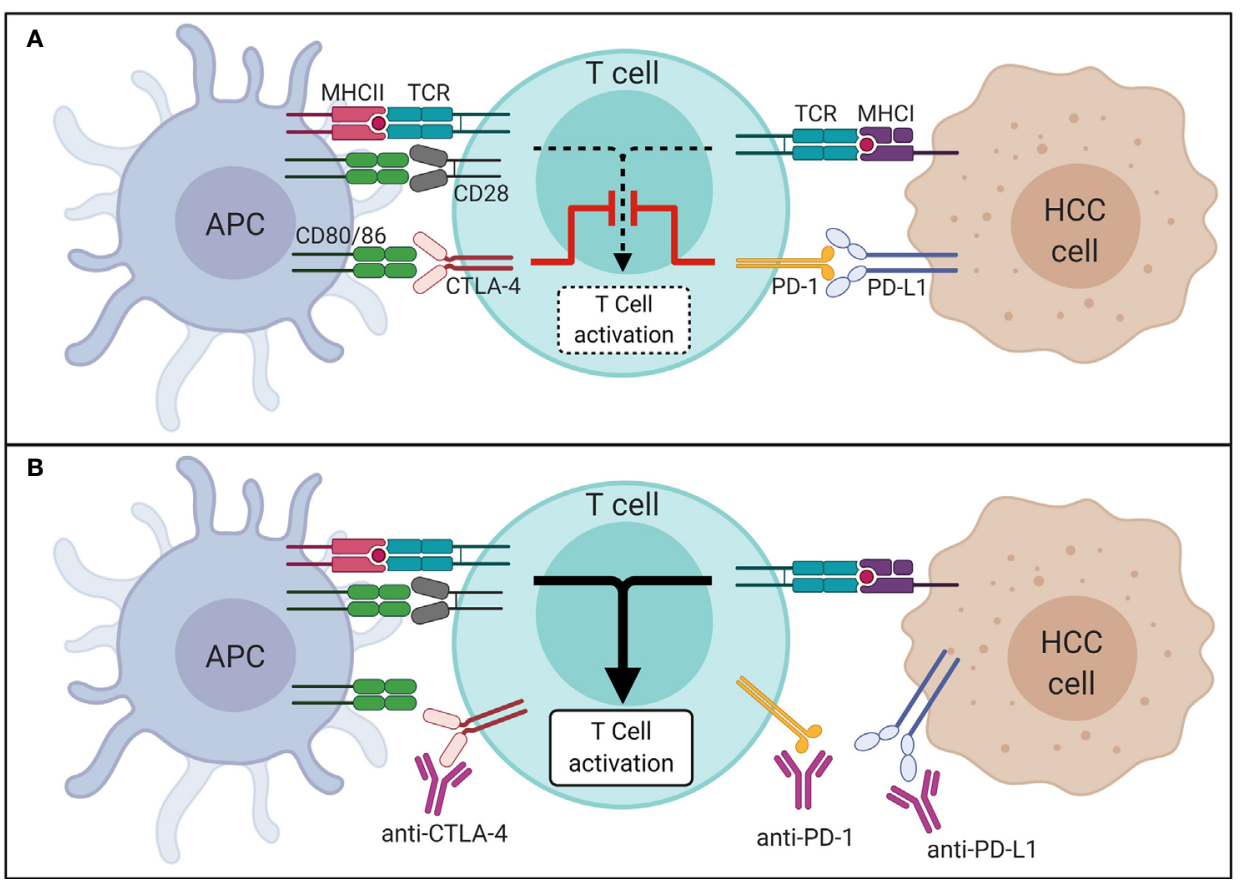

FIGURE 1 | Mechanism of immune checkpoints in HCC in absence (A) and presence (B) of ICl. APC, antigen presenting cell; CTLA-4, cytotoxic T-lymphocyteassociated protein 4; HCC, hepatocellular carcinoma; ICI, immune checkpoint inhibitor; MHC, major histocompatibility complex; PD-1, programmed cell death 1 protein; PD-L1, programmed death-ligand 1; TCR, T cell receptor. 
To establish potential associations between HCC immunobiology and clinical outcomes, inflammatory gene expression signatures were assessed retrospectively from the CheckMate 040 population (24). Tumor responses were observed regardless of tumor cell PD-L1 status. Median OS was 28.1 vs. 16.6 months for patients with tumor PD-L1 $\geq 1 \%$ vs. $<1 \%$ ( $\mathrm{p}=0.03$ ). Tumor inflammation measured by CD3 or CD8 showed a nonsignificant trend toward improved OS $(p=0.08)$, whereas macrophage markers were not associated with OS. Tumor PD-1 and PD-L1 expression were associated with improved OS ( $\mathrm{p}=0.05$ and $\mathrm{p}=0.03$, respectively). These analyses suggest that anti-tumor immune response may play a role in the treatment benefit of nivolumab in HCC.

In the keynote-224 (phase II, $\mathrm{n}=104$ ) and keynote-240 study (phase III, $n=413$ ) the PD-1 inhibitor pembrolizumab was applied after sorafenib failure or intolerance. Patients received a fixed dose of $200 \mathrm{mg}$ every 3 weeks (Q3W). In the phase II trial ORR (primary end point) was $18 \%$, DCR was $61 \%$, and OS was 12.9 months (25). The phase III trial compared pembrolizumab vs. placebo and failed to reach prespecified level of statistical significance for OS (13.9 vs. 10.6 months, respectively) and PFS (3.0 vs. 2.8 months, respectively) (26). Nevertheless, ORR was significantly higher with pembrolizumab $(18 \%$ vs. $4 \%, \mathrm{p}=0.00007)$, and median duration of response (DOR) was 13.8 months with pembrolizumab. Survival in the sorafenib control arm was again very long, attributable to the exclusion of macrovascular invasion, better management of patients, and the availability of subsequent therapies, including immunotherapies, that were not available at trial initiation (23). While failing statistical significance, a clinical benefit of durable responses for patients who achieved a response to treatment could be demonstrated in both studies.

Along with nivolumab and pembrolizumab, camrelizumab, another PD-1 antibody, was evaluated in a phase II trial with 3 $\mathrm{mg} / \mathrm{kg}$ every 2 or 3 weeks ( $\mathrm{n}=109$ vs. 108 , respectively). ORR was $15 \%$, OS probability at 6 months was $74 \%$, median OS was 13.8 months (27). Treatment-related serious adverse events occurred slightly higher in the every 2 weeks group (15\% vs. $7 \%)$. Immune-related adverse events of any cause occurred in $80 \%$ in the every 2 weeks group and $87 \%$ in the every 3 weeks group. Overall, camrelizumab had a safety profile similar to other PD-1 ICIs, except for higher occurrence of reactive cutaneous capillary endothelial proliferation.

Similar results were obtained when applying durvalumab $10 \mathrm{mg}$ / $\mathrm{kg}$ Q2W to pretreated HCC patients in a phase I/II trial $(\mathrm{n}=40)$. ORR was $10 \%$, median OS was 13.2 months (28).

In a phase Ia/Ib study tislelizumab's dose was evaluated with $200 \mathrm{mg}$ Q3W. ORR in pretreated HCC patients was 12\% (29). A phase III trial is comparing tislelizumab with sorafenib in treatment naïve patients, primary endpoint is OS (NCT03412773).

\section{COMBINATION STRATEGIES FOR IMMUNOTHERAPY}

Dual blockade of PD-(L)1 and VEGF has the potential to increase antitumoral activity through joint mechanisms
(30). This was the rationale for the phase $1 \mathrm{~b}$ study assessing efficacy and safety of atezolizumab $1200 \mathrm{mg}$ Q3W alone $(\mathrm{n}=59)$ and combined with bevacizumab $15 \mathrm{mg} / \mathrm{kg}$ Q3W $(\mathrm{n}=60)$ in a first line setting. Longer median PFS was associated with combination therapy compared to sole application of the ICI (5.6 vs. 3.4 months, $p=0.011$ ) (31). In the phase III IMbrave 150 trial a fixed dose of atezolizumab $1200 \mathrm{mg}$ and bevacizumab 15 $\mathrm{mg} / \mathrm{kg}$ Q3W $(\mathrm{n}=336)$ was compared with sorafenib $(\mathrm{n}=165)$ in a 2:1 ratio in therapy naïve patients with unresectable HCC and Child-Pugh score $\leq 6$. Coprimary endpoints were OS and PFS. Underlying etiology for liver cirrhosis was predominantly viral hepatitis B and C. Macrovascular invasion was frequent and most patients were staged as BCLC C. Median PFS was 6.8 months in the combination group and 4.3 months in the sorafenib group (HR: $0.59, \mathrm{p}<0.0001$ ). OS at 12 months was $67 \%$ vs. $55 \%$, respectively. Median OS was not reached in the combination arm. Grade 3 or 4 adverse events occurred in 57\% and 55\%, respectively. Except for hypertension, other high-grade toxic effects were infrequent (32). Besides symptoms of impaired liver function, patients with HCC frequently suffer from diverse conditions that limit their daily lives and make systemic therapy a challenge. Against this background, the effect on patients' quality of life is an increasingly important endpoint in the contemplation and evaluation of new therapies. The IMbrave 150 trial included the prespecified endpoints of time to deterioration (TTD) of quality of life, physical functioning, and role functioning, assessed by the European Organization for the Research and Treatment of Cancer Quality-of-Life Questionnaire (EORTC QLQ-C30). EORTC QLQ-C30 addresses these issues on a 100-point scale, with a drop of at least 10 points considered to be clinically meaningful (33). In both arms $>90 \%$ of patients completed the questionnaire, highlighting the quality of the analysis. Compared with sorafenib, the combination of atezolizumab/bevacizumab delayed TTD of patient-reported QOL (median TTD 11.2 vs. 3.6 months; physical functioning (median TTD 13.1 vs. 4.9 months), role functioning (median TTD 9.1 vs. 3.6 months)). Moreover, immunotherapy delayed TTD in patient-reported appetite loss, fatigue, pain, and diarrhea when comparing to sorafenib. A lower proportion of patients receiving the combination therapy experienced clinically meaningful deterioration in each of these symptoms when compared to TKI. In line with these results, a recent analysis demonstrated that the combination therapy showed similar efficacy regardless of age (34). In older patients, aged $\geq 65$ years, the median OS was not reached in the atezolizumab/bevacizumab arm vs. 14.9 months in the sorafenib arm. In older patients PFS (7.7 vs. 4.8 months, respectively) and ORR (26\% vs. $13 \%$, respectively) also favor the application of combination therapy. Frequency and severity of adverse events were similar between the 2 age groups and consistent with the known safety profiles of atezolizumab/ bevacizumab. Notably, no additional risks or toxicities were reported in older patients. Considering safety and efficacy data, these findings support an overall clinical benefit in patients with unresectable HCC. The combination of atezolizumab/ bevacizumab was recently approved by European authorities 
and is being incorporated in guidelines as first-line therapy in advanced HCC.

Another strategy to induce a stronger immune response and enhance the clinical efficacy of ICI monotherapy, was to simultaneously block two different immune checkpoints. In the setting of non-small-cell lung carcinoma and melanoma high doses of anti CTLA- 4 in combination with a PD-(L)1 inhibitor resulted in an initial proliferation and increase of peripheral $\mathrm{T}$ cells $(35,36)$. In the phase I/II CheckMate 040 trial nivolumab (anti PD-1) and ipilimumab (anti CTLA-4) were administrated in different doses and regimens to patients previously treated with sorafenib $(n=148)$. The primary endpoint ORR was $31 \%$ with a median DOR of 17 months. Thus, this combination led to an ORR twice that of nivolumab monotherapy. DCR was $49 \%$, OS at 24 months was $40 \%$ (37). These results led to the currently recruiting phase III CheckMate 9DW trial, comparing nivolumab $1 \mathrm{mg} / \mathrm{kg}$ Q3W plus ipilimumab $3 \mathrm{mg} / \mathrm{kg}$ for 4 doses to sorafenib or lenvatinib in therapy naïve patients with a ChildPugh sore $\leq 6$ (NCT04039607, planned $\mathrm{n}=1084)$. Primary endpoint is OS, secondary endpoints are ORR, DOR, and time to symptom deterioration (TTSD). In the phase I/II Study 22 trial patients with sorafenib failure or intolerance received durvalumab (anti PD-L1) and/or tremelimumab (anti CTLA-4) either as monotherapy or as combination therapy with different dosages $(n=40+332)$. Best median OS with 18.7 months could be achieved with the combination of a single priming dose of tremelimumab $300 \mathrm{mg}$ combined with durvalumab $1500 \mathrm{mg}$ being continued in a Q4W regimen, the ORR was 24\% (38). Pharmacodynamic biomarker analyses showed that CD8+ lymphocyte expansion was associated with treatment response. The durvalumab/tremelimumab combination is currently being compared to sorafenib in the phase III Himalaya trial in a first line setting (NCT03298451, planned $n=1200$ ). Primary endpoint is OS, secondary endpoints are TTP (time to progression), PFS, ORR, DCR, DOR, and safety.

A synergistic effect is expected, when combining immunotherapy and directly targeting TKIs. The phase Ibkeynote-524 trial tested lenvatinib (12 $\mathrm{mg}$ if $\geq 60 \mathrm{~kg}, 8 \mathrm{mg}$ if $<60 \mathrm{~kg}$ ) plus pembrolizumab 200 mg Q3W ( $\mathrm{n}=104)$. ORR was $46 \%$ with a median DOR of 12.6 months. Median PFS was 8.6 months (39). Based on these findings, a double-blind randomized phase III trial is comparing the combination of lenvatinib/pembrolizumab vs. lenvatinib alone in therapy naïve patients with Child-Pugh score A (NCT03713593, planned $n=750$ ). Primary endpoints are OS and PFS, secondary endpoints are ORR, DOR, DCR, TTP, and safety.

Other combinations are being tested in phase III trials in the setting of patients with advanced HCC who did not previously receive systemic therapy, e.g., atezolizumab/cabozantinib vs. sorafenib (NCT03755791, planned $\mathrm{n}=740$ ), or camrelizumab/ apatinib vs. sorafenib (NCT03764293, planned $n=510$ ). In this context, another CheckMate 040 cohort compared nivolumab/ cabozantinib vs. the triple combination of nivolumab/ipilimumab/ cabozantinib applied in different regimens. Investigator-assessed ORR was $17 \%$ in the nivolumab/cabozantinib arm and $26 \%$ in the nivolumab/ipilimumab/cabozantinib arm. DCR was $81 \%$ vs. $83 \%$, and median PFS was 5.5 vs. 6.8 months, respectively. Median
OS was not reached in either arm. No new safety signals were observed in either arm, demonstrating than even very intensive combinations are feasible in patients with HCC (40).

As described above, the concept of combination therapies is to increase the efficacy of ICI by further stimulating the immune response, meaning to "make cold tumors hot". Apart from pharmacological combinations, locoregional therapies (LRT) or transarterial chemoembolization (TACE) can be combination partners in this context. Besides local tumor control, they affect tumor immunity through complex mechanisms (41). LRT and TACE cause immunogenic cell death leading to the release of various tumor antigens. Moreover, they were demonstrated to enhance the number of dendritic cells in the HCC tumor microenvironment (42), leading to an increased antigen presentation and an enhanced response due to the activation of T-cells (43). Corroborating this concept, different trials are ongoing which are summarized in Table $\mathbf{1}$.

With autoimmune related adverse events (irAE), ICI therapy brought a novel spectrum of side effects, that was completely different than that known from chemotherapies. Risks of irAEs were widely reported as manageable and toxicity rates were generally lower than in TKI groups. Nevertheless, compared with cytotoxic agents, the possibility of identifying clinically relevant toxicity of ICI in early-phase clinical trials is relatively low (43\% vs. $70 \%$ ) (44). irAEs may develop long after the typical period of safety evaluation in oncology trials, and rather small sample sizes may not detect rare but life-threatening toxicity. In the IMbrave 150 study bleeding complications were observed in $7 \%$ of the atezolizumab/bevacizumab group vs. $4.5 \%$ in the sorafenib group. Although bleeding risk was not increased compared with that observed in previous anti-VEGF trials, a careful hepatologic management is necessary. The safety of ICI in the setting of advanced cirrhosis and their efficacy in different etiologies of liver injury remain open questions and need to be addressed in future trials.

\section{IMMUNOTHERAPY IN A (NEO) ADJUVANT SETTING}

HCC resection is in most cases not a definitive cure of malignancy, as recurrence rate after hepatectomy is high (45). Tumor recurrence after HCC resection is approximately $70 \%$ within 5 years, whereas up to $50 \%$ show early recurrence within the first 2 years, which is associated with tumor characteristics such as a large tumor, an incomplete tumor capsule, and venous or microvascular invasion (46). Nevertheless, neoadjuvant or adjuvant therapies are not recommended as they have not been proven to improve the outcome. The phase III Storm trial evaluated sorafenib as an adjuvant treatment, but concluded that it is not an effective intervention in such a setting (47). Therefore, adjuvant strategies in patients with HCC remain an unmet medical need.

Characteristics of the immune contexture have been shown to correlate with recurrence and outcome. The density of CD3 and CD8 T cells in the tumor and its margins is a prognostic marker for recurrence (48). The presence of T cells and cytotoxic cells as well as 
TABLE 1 | Ongoing clinical trials of immune checkpoint inhibitors (ICI) in combination with locoregional therapies (LRT) or transarterial chemoembolization (TACE).

\begin{tabular}{|c|c|c|c|c|c|c|}
\hline ICl & LRT/TACE & $\mathbf{N}$ & $\begin{array}{l}\text { Primary } \\
\text { Outcome }\end{array}$ & Secondary Outcome & Identifier (Name) & Phase \\
\hline durvalumab \pm bevacizumab & TACE & 600 & PFS & OS, QoL, & $\begin{array}{l}\text { NCT03778957 } \\
\text { (EMERALD-1) }\end{array}$ & III \\
\hline pembrolizumab + lenvatinib & TACE & 950 & PFS, OS & ORR, DCR, DOR, TTP, safety & & III \\
\hline PD-1 mAb, lenvatinib & TACE & 56 & ORR & PFS, TTP, DCR, DOR, OS & $\begin{array}{l}\text { NCT04273100 } \\
\text { (PLTHCC) }\end{array}$ & $\|$ \\
\hline camrelizumab & TACE & 60 & PFS & TTP, OS, ORR, DCR, DOR, safety & NCT04483284 & $\|$ \\
\hline durvalumab + tremelimumab & cryoablation, RFA, TACE & 50 & PFS & safety & NCT02821754 & $\|$ \\
\hline durvalumab + tremelimumab & radiation & 70 & ORR & safety, OS, DCR, PFS, DOR, TTP & NCT03482102 & $\|$ \\
\hline durvalumab + tremelimumab & Y-90 SIRT, TACE & 84 & ORR & PFS, OS, safety, ORR, QoL & $\begin{array}{l}\text { NCT04522544 } \\
\text { (IMMUWIN) }\end{array}$ & $\|$ \\
\hline nivolumab & TACE & 49 & ORR & PFS, TTP, OS, DOR, TTFS, QoL & $\begin{array}{l}\text { NCT03572582 } \\
\text { (IMMUTACE) }\end{array}$ & $\|$ \\
\hline nivolumab & Y-90 SIRT & 40 & ORR & $\begin{array}{l}\text { TTR, DOR, TTP, PFS, OS, QoL, } \\
\text { safety }\end{array}$ & NCT03033446 & $\|$ \\
\hline pembrolizumab & $\begin{array}{l}\text { RFA, MWA, brachytherapy, } \\
\text { TACE }\end{array}$ & 30 & ORR & TTR, RFS, OS, safety, biomarkers & NCT03753659 & $\|$ \\
\hline nivolumab & deb-TACE & 14 & safety & - & NCT03143270 & 1 \\
\hline pembrolizumab & TACE & 26 & safety & PFSR & NCT03397654 & $\mathrm{lb}$ \\
\hline
\end{tabular}

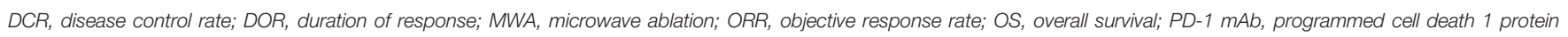

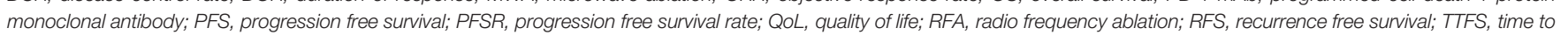
failure of strategy; TTP, time to progression; TTR, time to response.

the absence of macrophages and Th2 cells positively correlates with patient survival and does not differ between different etiologies and HCC stages (49). High expression of PD-L1 by tumor or immune cells is associated with a more aggressive tumor and is a predictor of recurrence (50). Altogether, there is a strong rationale for adjuvant immunotherapy and several clinical trials are investigating the role of ICI and antiangiogenic agents in an adjuvant setting. An overview of ongoing clinical trials is given in Table 2 .

ICI have the potential to achieve significantly higher ORR than TKI. Better tumor responses lead to tumor size reduction and may make secondary resectability feasible. Thus, ICI based regimens may even open the field of neoadjuvant strategies for HCC. The combination of ipilimumab and nivolumab is currently being evaluated as a neoadjuvant therapy in patients undergoing hepatic resection, assessing tumor shrinkage and OS after resection (see Table 2 ).

\section{CONCLUSION AND FUTURE DIRECTIONS}

Although ICI monotherapy could achieve good responses in some patients, they could not demonstrate superiority to TKI based therapies. Most recently, the atezolizumab/bevacizumab combination was associated with an unparalleled benefit of survival. Thus, immunotherapy is likely to have a huge impact on the management of HCC due to the ability to produce durable and clinically relevant responses. With the combination of different agents, higher response rates and longer overall survival may be achieved. Nevertheless, a significant percentage of HCC do not respond to immunotherapy and an immunologic classification is urgently needed to guide treatment decisions. In both CheckMate 040 and CheckMate 459, PD-L1 expression was not correlated with tumor response and patient prognosis (24). Experimental markers such as circulating tumor cells, cell free DNA, miRNA have been studied in the context of HCC (51). However, these do not currently play a role in routine clinical practice.

ICI brought a novel spectrum of immune related adverse events. Dual blockade of CTLA-4 and PD-1 or PD-L1 results in enhanced toxicities, especially when higher doses of CTLA-4 inhibitors are used. Combinations with TKI or anti-VEGF carry the risk of higher toxicities in cirrhotic patients that demand a close surveillance and hepatologic management. The selection of patients is crucial regarding safety. Alternative strategies than immunotherapy may be preferred in the setting of liver

TABLE 2 | Ongoing clinical trials of (neo)adjuvant immunotherapy for hepatocellular carcinoma (HCC).

\begin{tabular}{|c|c|c|c|c|}
\hline ICl & Controls & Setting & Identifier (Name) & Phase \\
\hline atezolizumab + bevacizumab & active surveillance & adjuvant after curative resection or ablation & NCT04102098 (IMbrave 050) & III \\
\hline durvalumab \pm bevacizumab & placebo & adjuvant after curative resection or ablation & NCT03847428 (EMERALD-2) & III \\
\hline nivolumab & placebo & adjuvant after curative resection or ablation & NCT03383458 (CheckMate 9DX) & III \\
\hline pembrolizumab & placebo & adjuvant after curative resection or ablation & NCT03867084 (KEYNOTE-937) & III \\
\hline nivolumab \pm ipilimumab & - & perioperative in (potentially) resectable HCC & NCT03222076 & $\|$ \\
\hline nivolumab + ipilimumab & - & neoadjuvant prior to resection & NCT03510871 & $\|$ \\
\hline nivolumab + ipilimumab & - & neoadjuvant prior to resection & NCT03682276 (PRIME-HCC) & $|/| \mid$ \\
\hline nivolumab + cabozantinib & - & neoadjuvant prior to resection & NCT03299946 & I \\
\hline
\end{tabular}


transplantation or uncontrolled autoimmune disease. Nevertheless, ICI have the potential to stabilize quality of life for patients with HCC. A longer time to deterioration of healthrelated quality of life was demonstrated under ICI when compared to TKI (33). To reach optimal benefit of immunotherapy, biomarkers to predict response are urgently needed. Furthermore, the therapeutic sequence of different classes and the combination of available agents needs to be identified. Despite all remaining challenges, checkpoint

\section{REFERENCES}

1. Singal AG, Lampertico P, Nahon P. Epidemiology and surveillance for hepatocellular carcinoma: New trends. J Hepatol (2020) 72(2):250-61. doi: 10.1016/j.jhep.2019.08.025

2. McGlynn KA, Petrick JL, El-Serag HB. Epidemiology of Hepatocellular Carcinoma. Hepatology (2021) 73 Suppl 1(Suppl 1):4-13. doi: 10.1002/ hep. 31288

3. Arnold M, Abnet CC, Neale RE, Vignat J, Giovannucci EL, McGlynn KA, et al. Global Burden of 5 Major Types of Gastrointestinal Cancer. Gastroenterology (2020) 159(1):335-349 e315. doi: 10.1053/j.gastro.2020.02.068

4. Fujiwara N, Friedman SL, Goossens N, Hoshida Y. Risk factors and prevention of hepatocellular carcinoma in the era of precision medicine. J Hepatol (2018) 68(3):526-49. doi: 10.1016/j.jhep.2017.09.016

5. Shiels MS, O'Brien TR. Recent Decline in Hepatocellular Carcinoma Rates in the United States. Gastroenterology (2020) 158(5):1503-5.e1502. doi: 10.1053/ j.gastro.2019.12.030

6. Chang MH, You SL, Chen CJ, Liu CJ, Lai MW, Wu TC, et al. Long-term Effects of Hepatitis B Immunization of Infants in Preventing Liver Cancer. Gastroenterology (2016) 151(3):472-480 e471. doi: 10.1053/j.gastro.2016.05.048

7. Forner A, Reig M, Bruix J. Hepatocellular carcinoma. Lancet (2018) 391 (10127):1301-14. doi: 10.1016/S0140-6736(18)30010-2

8. Pinter M, Scheiner B, Peck-Radosavljevic M. Immunotherapy for advanced hepatocellular carcinoma: a focus on special subgroups. Gut (2021) 70 (1):204-14. doi: 10.1136/gutjnl-2020-321702

9. Llovet JM, Ricci S, Mazzaferro V, Hilgard P, Gane E, Blanc JF, et al. Sorafenib in advanced hepatocellular carcinoma. N Engl J Med (2008) 359(4):378-90. doi: 10.1056/NEJMoa0708857

10. Llovet JM, Montal R, Sia D, Finn RS. Molecular therapies and precision medicine for hepatocellular carcinoma. Nat Rev Clin Oncol (2018) 15 (10):599-616. doi: 10.1038/s41571-018-0073-4

11. Raoul JL, Kudo M, Finn RS, Edeline J, Reig M, Galle PR. Systemic therapy for intermediate and advanced hepatocellular carcinoma: Sorafenib and beyond. Cancer Treat Rev (2018) 68:16-24. doi: 10.1016/j.ctrv.2018.05.006

12. Kudo M. Lenvatinib in Advanced Hepatocellular Carcinoma. Liver Cancer (2017) 6: (4):253-63. doi: 10.1159/000479573

13. Bi F, Qin S, Gu S, Bai Y, Chen Z, Wang Z, et al. Donafenib versus sorafenib as first-line therapy in advanced hepatocellular carcinoma: An open-label, randomized, multicenter phase II/III trial. J Clin Oncol (2020) 38 (15_suppl):4506-6. doi: 10.1200/JCO.2020.38.15_suppl.4506

14. Durante C, Russo D, Verrienti A, Filetti S. XL184 (cabozantinib) for medullary thyroid carcinoma. Expert Opin Investig Drugs (2011) 20(3):40713. doi: $10.1517 / 13543784.2011 .559163$

15. Bruix J, Qin S, Merle P, Granito A, Huang YH, Bodoky G, et al. Regorafenib for patients with hepatocellular carcinoma who progressed on sorafenib treatment (RESORCE): a randomised, double-blind, placebo-controlled, phase 3 trial. Lancet (2017) 389(10064):56-66. doi: 10.1016/S0140-6736(16)32453-9

16. Abou-Alfa GK, Meyer T, Cheng AL, El-Khoueiry AB, Rimassa L, Ryoo BY, et al. Cabozantinib in Patients with Advanced and Progressing Hepatocellular Carcinoma. N Engl J Med (2018) 379(1):54-63. doi: 10.1056/NEJMoa1717002

17. Zhu AX, Kang YK, Yen CJ, Finn RS, Galle PR, Llovet JM, et al. Ramucirumab after sorafenib in patients with advanced hepatocellular carcinoma and increased alpha-fetoprotein concentrations (REACH-2): a randomised, double-blind, placebo-controlled, phase 3 trial. Lancet Oncol (2019) 20 (2):282-96. doi: 10.1016/S1470-2045(18)30937-9 inhibitors have already today revolutionized the treatment of HCC.

\section{AUTHOR CONTRIBUTIONS}

All authors drafted the manuscript and provided intellectual input. All authors contributed to the article and approved the submitted version.

18. Ringelhan M, Pfister D, O'Connor T, Pikarsky E, Heikenwalder M. The immunology of hepatocellular carcinoma. Nat Immunol (2018) 19(3):222-32. doi: 10.1038/s41590-018-0044-z

19. El-Khoueiry AB, Sangro B, Yau T, Crocenzi TS, Kudo M, Hsu C, et al. Nivolumab in patients with advanced hepatocellular carcinoma (CheckMate 040): an open-label, non-comparative, phase $1 / 2$ dose escalation and expansion trial. Lancet (2017) 389(10088):2492-502. doi: 10.1016/S01406736(17)31046-2

20. Kudo M, Matilla A, Santoro A, Melero I, Gracian AC, Acosta-Rivera M, et al. Checkmate-040: Nivolumab (NIVO) in patients (pts) with advanced hepatocellular carcinoma (aHCC) and Child-Pugh B (CPB) status. J Clin Oncol (2019) 37(4_suppl):327-7. doi: 10.1200/JCO.2019.37.4_suppl.327

21. Sangro B, Park J, Finn R, Cheng A, Mathurin P, Edeline J, et al. LBA-3 CheckMate 459: Long-term (minimum follow-up 33.6 months) survival outcomes with nivolumab versus sorafenib as first-line treatment in patients with advanced hepatocellular carcinoma. Ann Oncol (2020) 31:S241-2. doi: 10.1016/j.annonc.2020.04.078

22. Yau T, Park JW, Finn RS, Cheng AL, Mathurin P, Edeline J, et al. LBA38_PR CheckMate 459: A randomized, multi-center phase III study of nivolumab (NIVO) vs sorafenib (SOR) as first-line (1L) treatment in patients (pts) with advanced hepatocellular carcinoma (aHCC). Ann Oncol (2019) 30:v874-5. doi: 10.1093/annonc/mdz394.029

23. Weinmann A, Galle PR. Role of immunotherapy in the management of hepatocellular carcinoma: current standards and future directions. Curr Oncol (2020) 27(Suppl 3):S152-64. doi: 10.3747/co.27.7315

24. Sangro B, Melero I, Wadhawan S, Finn RS, Abou-Alfa GK, Cheng AL, et al. Association of inflammatory biomarkers with clinical outcomes in nivolumab-treated patients with advanced hepatocellular carcinoma. J Hepatol (2020) 73(6):1460-9. doi: 10.1016/j.jhep.2020.07.026

25. Zhu AX, Finn RS, Edeline J, Cattan S, Ogasawara S, Palmer D, et al. Pembrolizumab in patients with advanced hepatocellular carcinoma previously treated with sorafenib (KEYNOTE-224): a non-randomised, open-label phase 2 trial. Lancet Oncol (2018) 19(7):940-52. doi: 10.1016/ S1470-2045(18)30351-6

26. Finn RS, Ryoo BY, Merle P, Kudo M, Bouattour M, Lim HY, et al. Pembrolizumab As Second-Line Therapy in Patients With Advanced Hepatocellular Carcinoma in KEYNOTE-240: A Randomized, Double-Blind, Phase III Trial. J Clin Oncol (2020) 38(3):193-202. doi: 10.1200/JCO.19.01307

27. Qin S, Ren Z, Meng Z, Chen Z, Chai X, Xiong J, et al. Camrelizumab in patients with previously treated advanced hepatocellular carcinoma: a multicentre, open-label, parallel-group, randomised, phase 2 trial. Lancet Oncol (2020) 21(4):571-80. doi: 10.1016/S1470-2045(20)30011-5

28. Wainberg ZA, Segal NH, Jaeger D, Lee K-H, Marshall J, Antonia SJ, et al. Safety and clinical activity of durvalumab monotherapy in patients with hepatocellular carcinoma (HCC). J Clin Oncol (2017) 35(15_suppl):4071-1. doi: 10.1200/JCO.2017.35.15_suppl.4071

29. Desai J, Deva S, Lee JS, Lin CC, Yen CJ, Chao Y, et al. Phase IA/IB study of single-agent tislelizumab, an investigational anti-PD-1 antibody, in solid tumors. J Immunother Cancer (2020) 8(1):e000453. doi: 10.1136/jitc-2019000453

30. Voron T, Colussi O, Marcheteau E, Pernot S, Nizard M, Pointet AL, et al. VEGF-A modulates expression of inhibitory checkpoints on CD8+ T cells in tumors. J Exp Med (2015) 212(2):139-48. doi: 10.1084/jem.20140559

31. Lee MS, Ryoo BY, Hsu CH, Numata K, Stein S, Verret W, et al. Atezolizumab with or without bevacizumab in unresectable hepatocellular carcinoma 
(GO30140): an open-label, multicentre, phase 1b study. Lancet Oncol (2020) 21(6):808-20. doi: 10.1016/S1470-2045(20)30156-X

32. Finn RS, Qin S, Ikeda M, Galle PR, Ducreux M, Kim TY, et al. Atezolizumab plus Bevacizumab in Unresectable Hepatocellular Carcinoma. N Engl J Med (2020) 382(20):1894-905. doi: 10.1056/NEJMoa1915745

33. Galle PR, Finn RS, Qin S, Ikeda M, Zhu AX, Kim T-Y, et al. Patient-reported outcomes (PROs) from the Phase III IMbrave150 trial of atezolizumab (atezo) + bevacizumab (bev) vs sorafenib (sor) as first-line treatment (tx) for patients (pts) with unresectable hepatocellular carcinoma (HCC). J Clin Oncol (2020) 38(4_suppl):476-6. doi: 10.1200/JCO.2020.38.4_suppl.476

34. Li D, Toh H, Merle P, Tsuchiya K, Hernandez S, Shao H, et al. O-8 Atezolizumab + bevacizumab vs sorafenib for unresectable hepatocellular carcinoma: Results from older adults enrolled in IMbrave150. Ann Oncol (2020) 31:234. doi: 10.1016/j.annonc.2020.04.061

35. Antonia S, Goldberg SB, Balmanoukian A, Chaft JE, Sanborn RE, Gupta A, et al. Safety and antitumour activity of durvalumab plus tremelimumab in non-small cell lung cancer: a multicentre, phase $1 \mathrm{~b}$ study. Lancet Oncol (2016) 17(3):299-308. doi: 10.1016/S1470-2045(15)00544-6

36. de Souza P, Malczewski A, Proscurshim I, Yuan G, Coart E, Dupont C, et al. Abstract CT104: Evaluation of peripheral $\mathrm{T}$ cell subset proliferation as a pharmacodynamic assay to guide the development of anti-CTLA-4 and PD-1 antibody combinations in patients with solid tumors. Cancer Res (2018) 78: CT104-4. doi: 10.1158/1538-7445.AM2018-CT104

37. Yau T, Kang Y-K, Kim T-Y, El-Khoueiry AB, Santoro A, Sangro B, et al. Nivolumab (NIVO) + ipilimumab (IPI) combination therapy in patients (pts) with advanced hepatocellular carcinoma (aHCC): Results from CheckMate 040. J Clin Oncol (2019) 37(15_suppl):4012-2.

38. Kelley RK, Sangro B, Harris WP, Ikeda M, Okusaka T, Kang Y-K, et al. Efficacy, tolerability, and biologic activity of a novel regimen of tremelimumab (T) in combination with durvalumab (D) for patients (pts) with advanced hepatocellular carcinoma (aHCC). J Clin Oncol (2020) 38(15_suppl):4508-8. doi: 10.1200/JCO.2020.38.15_suppl.4508

39. Finn RS, Ikeda M, Zhu AX, Sung MW, Baron AD, Kudo M, et al. Phase Ib Study of Lenvatinib Plus Pembrolizumab in Patients With Unresectable Hepatocellular Carcinoma. J Clin Oncol (2020) 38(26):2960-70. doi: 10.1200/JCO.20.00808

40. Yau T, Zagonel V, Santoro A, Acosta-Rivera M, Choo SP, Matilla A, et al. Nivolumab (NIVO) + ipilimumab (IPI) + cabozantinib (CABO) combination therapy in patients (pts) with advanced hepatocellular carcinoma (aHCC): Results from CheckMate 040. J Clin Oncol (2020) 38(4_suppl):478-8. doi: 10.1200/JCO.2020.38.4_suppl.478

41. Greten TF, Mauda-Havakuk M, Heinrich B, Korangy F, Wood BJ. Combined locoregional-immunotherapy for liver cancer. J Hepatol (2019) 70(5):9991007. doi: 10.1016/j.jhep.2019.01.027

42. den Brok MH, Sutmuller RP, Nierkens S, Bennink EJ, Frielink C, Toonen LW, et al. Efficient loading of dendritic cells following cryo and radiofrequency ablation in combination with immune modulation induces anti-tumour immunity. Br J Cancer (2006) 95(7):896-905. doi: 10.1038/sj.bjc.6603341
43. Singh P, Toom S, Avula A, Kumar V, Rahma OE. The Immune Modulation Effect of Locoregional Therapies and Its Potential Synergy with Immunotherapy in Hepatocellular Carcinoma. $J$ Hepatocell Carcinoma (2020) 7:11-7. doi: 10.2147/JHC.S187121

44. Beaver JA, Howie LJ, Pelosof L, Kim T, Liu J, Goldberg KB, et al. A 25-Year Experience of US Food and Drug Administration Accelerated Approval of Malignant Hematology and Oncology Drugs and Biologics: A Review. JAMA Oncol (2018) 4(6):849-56. doi: 10.1001/jamaoncol.2017.5618

45. Zheng J, Chou JF, Gonen M, Vachharajani N, Chapman WC, Majella Doyle MB, et al. Prediction of Hepatocellular Carcinoma Recurrence Beyond Milan Criteria After Resection: Validation of a Clinical Risk Score in an International Cohort. Ann Surg (2017) 266(4):693-701. doi: 10.1097/SLA.0000000000002360

46. Imamura $\mathrm{H}$, Matsuyama $\mathrm{Y}$, Tanaka E, Ohkubo $\mathrm{T}$, Hasegawa K, Miyagawa S, et al. Risk factors contributing to early and late phase intrahepatic recurrence of hepatocellular carcinoma after hepatectomy. J Hepatol (2003) 38(2):200-7. doi: 10.1016/S0168-8278(02)00360-4

47. Bruix J, Takayama T, Mazzaferro V, Chau GY, Yang J, Kudo M, et al. Adjuvant sorafenib for hepatocellular carcinoma after resection or ablation (STORM): a phase 3, randomised, double-blind, placebo-controlled trial. Lancet Oncol (2015) 16(13):1344-54. doi: 10.1016/S1470-2045(15)00198-9

48. Gabrielson A, Wu Y, Wang H, Jiang J, Kallakury B, Gatalica Z, et al. Intratumoral CD3 and CD8 T-cell Densities Associated with Relapse-Free Survival in HCC. Cancer Immunol Res (2016) 4(5):419-30. doi: 10.1158/2326-6066.CIR-15-0110

49. Foerster F, Hess M, Gerhold-Ay A, Marquardt JU, Becker D, Galle PR, et al. The immune contexture of hepatocellular carcinoma predicts clinical outcome. Sci Rep (2018) 8(1):5351. doi: 10.1038/s41598-018-21937-2

50. Calderaro J, Rousseau B, Amaddeo G, Mercey M, Charpy C, Costentin C, et al. Programmed death ligand 1 expression in hepatocellular carcinoma: Relationship With clinical and pathological features. Hepatology (2016) 64 (6):2038-46. doi: 10.1002/hep.28710

51. Labgaa I, Villanueva A, Dormond O, Demartines N, Melloul E. The Role of Liquid Biopsy in Hepatocellular Carcinoma Prognostication. Cancers (Basel) (2021) 13(4):659. doi: 10.3390/cancers13040659

Conflict of Interest: The authors declare that the research was conducted in the absence of any commercial or financial relationships that could be construed as a potential conflict of interest.

The reviewer PS declared a past collaboration with one of the authors FT to the handling editor.

Copyright (๑) 2021 Mohr, Jost-Brinkmann, Özdirik, Lambrecht, Hammerich, Loosen, Luedde, Demir, Tacke and Roderburg. This is an open-access article distributed under the terms of the Creative Commons Attribution License (CC BY). The use, distribution or reproduction in other forums is permitted, provided the original author(s) and the copyright owner(s) are credited and that the original publication in this journal is cited, in accordance with accepted academic practice. No use, distribution or reproduction is permitted which does not comply with these terms. 